\title{
Editorial: Magnetic
}

Resonance-Guided Focused

\section{Ultrasound: Physical Principles and Biomedical Applications}

\author{
Allegra Conti ${ }^{\star \star t}$, Hermes A. S. Kamimura ${ }^{2 \star t}$, Anthony Novell ${ }^{3}$, Andrea Duggento ${ }^{1}$ and \\ Nicola Toschi ${ }^{1,4}$
}

${ }^{1}$ Medical Physics Section, Department of Biomedicine and Prevention, University of Rome Tor Vergata, Rome, Italy, ${ }^{2}$ Department of Biomedical Engineering, Columbia University, New York, NY, United States, ${ }^{3}$ Université Paris-Saclay, CEA, CNRS, Inserm, BioMaps, Service Hospitalier Frédéric Joliot, Orsay, France, ${ }^{4}$ Athinoula A. Martinos Center for Biomedical Imaging, Harvard Medical School, Boston, MA, United States

Keywords: magnetic resonance imaging, focused ultrasound, magnetic resonance imaging-guided focused ultrasound (MRgFUS), focused ultrasound ablation, drug delivery system, blood-brain barrier opening, therapeutic ultrasound

\section{Editorial on the Research Topic}

Magnetic Resonance-Guided Focused Ultrasound: Physical Principles and Biomedical Applications

OPEN ACCESS

Edited and reviewed by:

Thomas Beyer,

Medical University of Vienna, Austria

*Correspondence:

Allegra Cont

allegra.conti@uniroma2.it

Hermes A. S. Kamimura

kamimura.hermes@columbia.edu

tThese authors have contributed equally to this work

Specialty section

This article was submitted to Medical Physics and Imaging,

a section of the journal Frontiers in Physics

Received: 22 March 2021 Accepted: 08 April 2021 Published: 14 May 2021

Citation:

Conti A, Kamimura HAS, Novell A,

Duggento A and Toschi N (2021)

Editorial: Magnetic Resonance-Guided

Focused Ultrasound: Physical

Principles and Biomedical

Applications. Front. Phys. 9:683899.

doi: 10.3389/fphy.2021.683899
Magnetic resonance-guided focused ultrasound (MRgFUS) is a non-invasive technique used in more than ninety clinical trials as an alternative to standard treatments for neurological, oncological and musculoskeletal diseases. Indeed, magnetic resonance (MR)-guidance allows targeted treatments through a wide range of focused ultrasound (FUS) intensities inducing mechanical, thermal, and neuroelectric effects on the tissue while preserving surrounding organs-at-risk. This Research Topic gathered contributions from experts in MRgFUS and summarized recent and impactful results supporting clinical and preclinical research obtained with high and low acoustic intensities regimes.

The application of MR-guidance in thermal ablations through high-intensity focused ultrasound (HIFU) is currently the subject of a number of studies and clinical trials aiming at treating medically refractory essential tremor, Parkinson's disease, neuropathic pain [1] as well as breast, liver, prostate, and brain cancers [2]. Gagliardo et al. present a retrospective analysis of patient- and sonication-related parameters in a group of patients undergoing unilateral ventral intermediate nucleus (Vim) thalamotomy through HIFU using a system integrated into a 1.5-T magnetic resonance imaging (MRI) unit. Their results confirm that the skull density ratio (SDR) is critical in determining thermal effects induced during sonication. Also, the authors suggest that energy deposition and SDR dependence need careful consideration and should be prescribed in a personalized manner.

At the other end of the acoustic spectrum, low-intensity focused ultrasound (LIFU) is frequently used under MR-guidance for locally and reversibly eliciting excitatory and inhibitory neuromodulation [3,4] or to facilitate drug and gene delivery through the permeabilization of the blood-brain barrier (BBB) [5-9]. The emergent idea of using FUS to modulate brain activity and neurovascular coupling demonstrates great potential to help understand brain functioning and treat brain diseases. Kamimura et al. describe the main potential mechanisms of ultrasound-based neuromodulation and how combining MRI with multimodal stimulation approaches can help advance the field. There are multiple hypotheses about the potential mechanisms through which ultrasound induces neuromodulation, mainly based on depolarization through mechanical 
deformation of the cell membrane and heat deposition. Other forms of ultrasound interactions with tissue can also be obtained with non-linear frequency mixing [10] generated by specifically designed transducers [11]. In this context, multimodal stimulation, coupled with neuroelectric or MRI, may provide an opportunity to understand the multiple factors that play a role in neuron functioning and how FUS interferes with it. In turn, this may elucidate the spatial and temporal scales of the mechanisms of action involved, the differential effect of FUS neuromodulation on different types of brain cells, and therefore the role of specific white matter pathways to neuronal network dynamics and animal behavior. In addition, a multimodal approach involving the combination of genetic, magnetic, ultrasonic, and other stimulation techniques to excite, inhibit, or regulate neuronal activity could be beneficial for both main symptoms and comorbidities. In turn, this may lead to patient-specific customization of neuromodulation interventions according to overall anamnestic picture. Furthermore, the use of MRI can provide insights into brain structure and activity and hence support FUS-based neuromodulation through targeting, safety evaluation, and the evaluation of brain function and mechanisms.

The intersection of engineering and health science enables the investigation of FUS's ability to interfere with brain activity in a safe, customized, and non-invasive manner. FUS can probe spatially specific brain regions non-invasively $[4,12]$, and in the case of peripheral nerves, the pulsing regime dictates excitatory or inhibitory effects [13-16]. Pouget et al. demonstrate that transcranial ultrasound stimulation using a repetitive pulse sequence can modulate the visuomotor behavior of nonhuman primates, demonstrating for the first time that repetitive transcranial ultrasound (rTUS) assisted by neuronavigation [17] can have a sustained effect on monkey behavior with a quantified return-time to baseline (18-31 $\mathrm{min})$.

Since its discovery in 2001, MR-guided LIFU-induced drug delivery has received widespread attention. LIFU, in conjunction with intravenously injected gas-encasing microbubbles (MBs), can reversibly disrupt the blood-brain barrier (BBB), hence allowing the targeted delivery of drugs or other active biological molecules in the brain. Conti et al. revise the state of the art in current MRI hardware and methods used in BBB opening protocols both in preclinical and clinical settings. The authors show that FUS systems developed for preclinical experiments are mostly constituted by single-element transducers, compatible with MRI field strength between 7T and 9.4T [8, 18, 19]. On the other hand, most clinical trials use a commercial 1024-element US phased array (INSIGHTEC, Haifa, Israel), which can be integrated into MRI scanners operating both at 1.5T and 3T [20]. These systems allow the delivery of nanometer-scale particles to the brain tissue $[21,22]$ to treat amyotrophic lateral sclerosis [23], Alzheimer's and Parkinson's disease [20, 24], as well as glioblastoma [25].

Cancer therapy can also be enhanced by applying MR-guided LIFU to deliver nanovectors capable of encapsulating drugs and releasing them following acoustic stimuli. Patrucco and Terreno present an overview of preclinical studies illustrating the in vivo potential of MRI-guided drug release protocols triggered by thermal and mechanical ultrasound-induced effects, where MRI is used to monitor release processes but also to evaluate therapeutic outcomes.

Microbubble specifications play a major role in LIFUinduced BBB disruption [26]. Originally designed as imaging contrast agents, microbubbles can be engineered specifically for therapeutic applications with increased stability and penetration into the blood or loaded with MRI contrast agents to image their biodistribution [27]. Also, microbubble characteristics such as size, concentration, gas and shell composition, dissolution, and clearance rate can influence BBB permeability and inflammatory response [28]. Pouliopoulos et al. describe an original study on the temporal stability of lipid-shelled microbubbles during therapeutic LIFU exposure. Storage time decreases in vitro microbubble stability, reducing stable cavitation response and promoting microbubble collapse. Considering the natural concentration decay of their microbubbles, the authors demonstrate that efficient $\mathrm{BBB}$ opening could be performed over a period of 3 weeks after homemade microbubble activation. These findings suggest that repeated treatments using stored microbubbles are possible for both pre-clinical and clinical applications over several weeks.

Although most parts of MR-compatible US systems are developed to exploit MR images for US targeting, planning and confirmation, MR-compatible acoustic instrumentation can also be used for developing multimodal imaging improving both spatial and temporal resolution of MR and ultrasound imaging [29]. Hasegawa et al. present a flexible-shape ultrasonic array probe suitable for ultrasonic imaging of the brain and compatible with high-field MR environments. This probe can be easily adapted to fit head shape while its curved-surface and, in turn, the coordinates of the elements can be employed to correct B-mode images.

In conclusion, this Research Topic illustrates different uses of MR-guidance for FUS-based therapeutic strategies. MR is mostly used to create treatment planning and confirm targeting. In contrast, US can be used in manifold ways, e.g., by exploiting high intensities for non-invasive surgery or by inducing through low intensity, transient modulation of vasculature permeability and neural activity.

The ever-increasing use of various MRgFUS techniques for surgery and drug delivery suggests that we will witness their swift introduction into novel experimental clinical paradigms, as recently demonstrated by novel HIFU application such as e.g., in treatment of brain tumors, stroke, epilepsy, pain, and functional disorders as well as by LIFU applications for immunotherapy, gene, and cell therapy [30,31].

\section{AUTHOR CONTRIBUTIONS}

AC, HK, NT, and AN made contributions about the papers they edited. All authors drafted and revised the manuscript. 


\section{REFERENCES}

1. Dobrakowski PP, Machowska-Majchrzak AK, Labuz-Roszak B, Majchrzak KG, Kluczewska E, Pierzchała KB. MR-guided focused ultrasound: a new generation treatment of Parkinson's disease, essential tremor and neuropathic pain. Interv Neuroradiol. (2014) 20:275-82. doi: 10.15274/INR-2014-10033

2. Hsiao YH, Kuo SJ, Tsai HD, Chou MC, Yeh GP. Clinical application of high-intensity focused ultrasound in cancer therapy. J Cancer. (2016) 7:22531. doi: $10.7150 /$ jca. 13906

3. Blackmore J, Shrivastava S, Sallet J, Butler CR, Cleveland RO. Ultrasound neuromodulation: a review of results, mechanisms and safety. Ultrasound Med Biol. (2019) 45:1509-1536. doi: 10.1016/j.ultrasmedbio.2018.12.015

4. Kamimura HAS, Wang S, Chen H, Wang Q, Aurup C, Acosta C, et al. Focused ultrasound neuromodulation of cortical and subcortical brain structures using 1.9 MHz. Med Phys. (2016) 43:5730. doi: 10.1118/1.4963208

5. Novell A, Kamimura HAS, Cafarelli A, Gerstenmayer M, Flament J, Valette J, et al. A new safety index based on intrapulse monitoring of ultraharmonic cavitation during ultrasound-induced blood-brain barrier opening procedures. Sci Rep. (2020) 10:10088. doi: 10.1038/s41598-020-66994-8

6. Conti A, Magnin R, Gerstenmayer M, Tsapis N, Dumont E, Tillement O, et al. Empirical and theoretical characterization of the diffusion process of different gadolinium-based nanoparticles within the brain tissue after ultrasoundinduced permeabilization of the blood-brain barrier. Contrast Media Mol Imag. (2019) 2019:6341545. doi: 10.1155/2019/6341545

7. Fisher DG, Price RJ. Recent advances in the use of focused ultrasound for magnetic resonance image-guided therapeutic nanoparticle delivery to the central nervous system. Front Pharmacol. (2019) 10:1348. doi: 10.3389/fphar.2019.01348

8. Kamimura HA, Flament J, Valette J, Cafarelli A, Aron Badin R, Hantraye P, et al. Feedback control of microbubble cavitation for ultrasoundmediated blood-brain barrier disruption in non-human primates under magnetic resonance guidance. J Cereb Blood Flow Metab. (2019) 39:1191203. doi: $10.1177 / 0271678 X 17753514$

9. Kamimura H, Wang S, Wu S-Y, Karakatsani M, Acosta C, Carneiro A, et al. Chirp- and random-based coded ultrasonic excitation for localized blood-brain barrier opening. Phys Med Biol. (2015) 60:7695712. doi: $10.1088 / 0031-9155 / 60 / 19 / 7695$

10. Baggio AL, Kamimura HAS, Henrique Lopes J, Carneiro AAO, Silva GT. Parametric array signal in confocal vibro-acoustography. Applied Acoustics. (2017) 126:143-8. doi: 10.1016/j.apacoust.2017.05.022

11. Kamimura HAS, Urban MW, Carneiro AAO, Fatemi M, Alizad A. Vibro-acoustography beam formation with reconfigurable arrays. IEEE Trans Ultrason Ferroelectr Freq Control. (2012) 59:1421-31. doi: 10.1109/TUFFC.2012.2343

12. Aurup C, Kamimura HAS, Konofagou EE. High-resolution focused ultrasound neuromodulation induces limb-specific motor responses in mice in vivo. Ultrasound Med Biol. (2021) 47:998-1013. doi: 10.1016/j.ultrasmedbio.2020.12.013

13. Downs ME, Lee SA, Yang G, Kim S, Wang Q, Konofagou EE. Non-invasive peripheral nerve stimulation via focused ultrasound in vivo. Phys Med Biol. (2018) 63:035011. doi: 10.1088/1361-6560/aa9fc2

14. Lee SA, Kamimura HAS, Burgess MT, Konofagou EE. Displacement imaging for focused ultrasound peripheral nerve neuromodulation. IEEE Trans Med Imaging. (2020) 39:3391-402. doi: 10.1109/TMI.2020.29 92498

15. Lee SA, Kamimura HAS, Konofagou EE. Displacement imaging during focused ultrasound median nerve modulation: a preliminary study in human pain sensation mitigation. IEEE Trans Ultrason Ferroelectr Freq Control. (2021) 68:526-37. doi: 10.1109/TUFFC.2020.3014183

16. Kim MG, Kamimura HAS, Lee SA, Aurup C, Kwon N, Konofagou EE. Imageguided focused ultrasound modulates electrically evoked motor neuronal activity in the mouse peripheral nervous system in vivo. J Neural Eng. (2020) 17:026026. doi: 10.1088/1741-2552/ab6be6

17. Wu S-Y, Aurup C, Sanchez CS, Grondin J, Zheng W, Kamimura H, et al. Efficient blood-brain barrier opening in primates with neuronavigationguided ultrasound and real-time acoustic mapping. Sci Rep. (2018) 8:7978. doi: 10.1038/s41598-018-25904-9
18. Magnin R, Rabusseau F, Salabartan F, Mériaux S, Aubry J-F, Le Bihan D, et al. Magnetic resonance-guided motorized transcranial ultrasound system for blood-brain barrier permeabilization along arbitrary trajectories in rodents. J Ther Ultrasound. (2015) 3:1-11. doi: 10.1186/s40349-015-0044-5

19. Choi JJ, Selert K, Gao Z, Samiotaki G, Baseri B, Konofagou EE. Noninvasive and localized blood-brain barrier disruption using focused ultrasound can be achieved at short pulse lengths and low pulse repetition frequencies. J Cereb Blood Flow Metab. (2011) 31:725-37. doi: 10.1038/jcbfm.2010.155

20. Lipsman N, Meng Y, Bethune AJ, Huang Y, Lam B, Masellis M, et al. Blood-brain barrier opening in Alzheimer's disease using MR-guided focused ultrasound. Nat Commun. (2018) 9:1-8. doi: 10.1038/s41467-018-04529-6

21. Conti A, Mériaux S, Larrat B. About the Marty model of blood-brain barrier closure after its disruption using focused ultrasound. Phys Med Biol. (2019) 64:1-6. doi: 10.1088/1361-6560/ab259d

22. Conti A, Magnin R, Gerstenmayer M, Lux F, Tillement O, Mériaux S, et al. Characterization of the diffusion process of different Gadoliniumbased nanoparticles within the brain tissue after ultrasound induced Blood-Brain Barrier permeabilization. In Proceedings of the 2016 IEEE International Ultrasonics Symposium (IUS) (Tours) (2016), 1-4. doi: 10.1109/ULTSYM.2016.7728773

23. Abrahao A, Meng Y, Llinas M, Huang Y, Hamani C, Mainprize T, et al. First-in-human trial of blood-brain barrier opening in amyotrophic lateral sclerosis using MR-guided focused ultrasound. Nat Commun. (2019) 10:19. doi: 10.1038/s41467-019-12426-9

24. Karakatsani ME, Blesa J, Konofagou EE. Blood-brain barrier opening with focused ultrasound in experimental models of Parkinson's disease. Mov Disord. (2019) 34:1252-61. doi: 10.1002/mds.27804

25. Idbaih A, Canney M, Belin L, Desseaux C, Vignot A, Bouchoux G, et al. Safety and feasibility of repeated and transient blood-brain barrier disruption by pulsed ultrasound in patients with recurrent glioblastoma. Clin Cancer Res. (2019) 25:3793-801. doi: 10.1158/1078-0432.CCR-18-3643

26. Dauba A, Delalande A, Kamimura HAS, Conti A, Larrat B, Tsapis N, et al. Recent advances on ultrasound contrast agents for blood-brain barrier opening with focused ultrasound. Pharmaceutics. (2020) 12:130. doi: 10.3390/pharmaceutics12111125

27. Liao AH, Liu HL, Su CH, Hua MY, Yang HW, Weng YT, et al. Paramagnetic perfluorocarbon-filled albumin-(Gd-DTPA) microbubbles for the induction of focused-ultrasound-induced blood-brain barrier opening and concurrent MR and ultrasound imaging. Phys Med Biol. (2012) 57:2787802. doi: 10.1088/0031-9155/57/9/2787

28. McMahon D, Lassus A, Gaud E, Jeannot V, Hynynen K. Microbubble formulation influences inflammatory response to focused ultrasound exposure in the brain. Scientific Reports. (2020) 10:21534. doi: 10.1038/s41598-020-78657-9

29. Curiel L, Chopra R, Hynynen K. Progress in multimodality imaging: truly simultaneous ultrasound and magnetic resonance imaging. IEEE Trans Med Imaging. (2007) 26:1740-6. doi: 10.1109/TMI.2007. 903572

30. Quadri SA, Waqas M, Khan I, Khan MA, Suriya SS, Farooqui M, et al. High-intensity focused ultrasound: past, present, and future in neurosurgery. Neurosurg Focus. (2018) 44:E16. doi: 10.3171/2017.11.FOCUS 17610

31. Stavarache MA, Chazen JL, Kaplitt MG. Innovative applications of MRguided focused ultrasound for neurological disorders. World Neurosurg. (2021) 145:581-9. doi: 10.1016/j.wneu.2020.08.052

Conflict of Interest: The authors declare that the research was conducted in the absence of any commercial or financial relationships that could be construed as a potential conflict of interest.

Copyright $\odot 2021$ Conti, Kamimura, Novell, Duggento and Toschi. This is an openaccess article distributed under the terms of the Creative Commons Attribution License (CC BY). The use, distribution or reproduction in other forums is permitted, provided the original author(s) and the copyright owner(s) are credited and that the original publication in this journal is cited, in accordance with accepted academic practice. No use, distribution or reproduction is permitted which does not comply with these terms. 